\title{
Dynamical Response of a Bose-Einstein Condensate to a Discontinuous Change in Internal State
}

\author{
M.R. Matthews, D.S. Hall, D.S. Jin, J.R. Ensher, C.E. Wieman, and E.A. Cornel䊝 \\ JILA and Department of Physics, University of Colorado and National Institute of Standards and Technology, Boulder, \\ Colorado 80309-0440 \\ F. Dalfovo, C. Minniti, and S. Stringari \\ Dipartimento di Fisica, Università di Trento, and Istituto Nazionale Fisica della Materia, I-38050 Povo, Italy
}

(March 2, 1998)

\begin{abstract}
A two-photon transition is used to convert an arbitrary fraction of the ${ }^{87} \mathrm{Rb}$ atoms in a $\left|F=1, m_{f}=-1\right\rangle$ condensate to the $\left|F=2, m_{f}=1\right\rangle$ state. Transferring the entire population imposes a discontinuous change on the condensate's mean-field repulsion, which leaves a residual ringing in the condensate width. A calculation based on Gross-Pitaevskii theory agrees well with the observed behavior, and from the comparison we obtain the ratio of the intraspecies scattering lengths for the two states, $a_{|1,-1\rangle} / a_{|2,1\rangle}=1.062(12)$.
\end{abstract}

The effects of interactions on dilute atomic BoseEinstein condensates (BEC) [1] have been studied in several contexts, including density and momentum distributions [2], collective excitations [3], specific heat [4], speed of sound [5], and limited condensate number [6]. There has been excellent quantitative agreement between these experiments and theory, made possible by both experimental advances and the fact that the interactions can be modeled relatively simply. Despite complicated interatomic potentials, the mean-field interaction in condensates is well-characterized by a single parameter, the $s$-wave scattering length $a$. Previous quantitative experiments on interactions have all been done using singlecomponent condensates with a constant value of $a$, but several authors have proposed using external optical or magnetic fields to shift the mean-field interaction by perturbing the interatomic potential [77 9].

We present a method for the creation of condensate mixtures using radiofrequency (rf) and microwave fields. We are able to transfer abruptly a trapped condensate of one hyperfine state into a coherent superposition of two trapped hyperfine states, and then watch the subsequent dynamical behavior. This approach makes possible a variety of two-species BEC studies. In this paper, we examine quantitatively one special case, in which all condensate atoms are converted from one state to another. Since these two states have slightly different values of $a$, the sudden change in self-interaction gives rise to oscillatory spatial behavior of the condensate wave function [10]. The scattering length ratio can be extracted from a model using analytical equations of motion for the con- densate widths 10 12] based on Gross-Pitaevskii theory.

The first demonstration of a binary mixture of condensates by Myatt et al. [13] produced overlapping condensates of the $5 S_{1 / 2}\left|F=1, m_{f}=-1\right\rangle$ and $\left|F=2, m_{f}=2\right\rangle$ states of ${ }^{87} \mathrm{Rb}$. The ratio of the magnetic moments of these states is $1: 2$, so the condensates experience different potentials in a magnetic trap and are displaced unequally from the trap center by gravity. Due to an accidental coincidence between the singlet and triplet scattering lengths of ${ }^{87} \mathrm{Rb}$, collisional loss is reduced and any mixture of spin states will be relatively long-lived 13 15. Here, we use mixtures of $|1,-1\rangle$ and $|2,1\rangle$ states, which possess several advantages. First, these two states have essentially identical magnetic moments, and hence feel identical confining potentials. Second, one can conveniently and quickly change atoms from the $|1,-1\rangle$ state to the $|2,1\rangle$ state by a two-photon transition (microwave plus rf). Finally, we can selectively image the different components using appropriately tuned lasers.

The apparatus uses a multiply loaded, double magneto-optical trap (MOT) scheme [16], consisting of two MOT regions connected by a $60 \mathrm{~cm}$ long, $1 \mathrm{~cm}$ diameter transfer tube. In the first MOT, ${ }^{87} \mathrm{Rb}$ atoms are collected from a background $\mathrm{Rb}$ vapor, produced by heating a Rb getter source 17]. The trapping beams are then turned off and a near-resonant laser beam is pulsed on to push the atoms through the transfer tube into the second MOT. To prevent collisions with the walls, the outside of the transfer tube is lined with strips of permanent magnetic material which form a hexapole guiding field. The atoms exit the tube and are captured by the second MOT, which has a 70 second lifetime due to differential pumping. This process is repeated twice a second for $\sim 10 \mathrm{~s}$ until approximately $10^{9}$ atoms have accumulated in the second MOT.

The atoms are further cooled in an optical molasses and then optically pumped into the $|1,-1\rangle$ state. These atoms are then transferred into a time-averaged, orbiting potential (TOP) magnetic trap [18]. The TOP trap consists of a quadrupole magnetic field with an axial gradient of $\sim 250 \mathrm{G} / \mathrm{cm}$ and a uniform magnetic field which rotates at $1.8 \mathrm{kHz}$. The fraction of atoms captured from molasses in the TOP trap depends approximately linearly on the magnitude of the rotating field. We use the maxi- 
mum rotating field achievable in our apparatus (49 G) to capture up to $50 \%$ of the atoms from the MOT, substantially enhancing the initial number as compared to our previous setup [1]. As in [1], the confined cloud is then magnetically compressed and evaporatively cooled to a temperature where there is no noticeable non-condensed fraction remaining, corresponding to a temperature less than 0.4 of the BEC transition temperature. Evaporative cooling requires about 30 seconds, and typically produces a condensate of $5 \times 10^{5}$ atoms.

The magnetic moments of the $|1,-1\rangle$ and $|2,1\rangle$ states are nearly the same to first order in magnetic field. Second-order dependence, the nuclear magnetic moment, and small effects due to the time varying nature of the TOP trap must be taken into account when calculating the exact trap potential 19,20 . The quadrupole and rotating magnetic fields are adiabatically changed after evaporation to make the trap potentials the same (to within $0.3 \%$ ) for the two states. Evaporation takes place in a trap with radial frequency $\nu_{r}=35 \mathrm{~Hz}$; subsequent work occurs at $\nu_{r}=17 \mathrm{~Hz}$ with a rotating field magnitude of $5.7 \mathrm{G}\left(\nu_{r}=\nu_{z} / \sqrt{8}\right.$ for the TOP trap).

The two-photon transition used to change the hyperfine state is shown schematically in Fig. 11a. We apply a pulse of microwave radiation at a frequency slightly less than the ground state hyperfine splitting of ${ }^{87} \mathrm{Rb}$ $(\sim 6.8 \mathrm{GHz})$ along with a $\sim 2 \mathrm{MHz}$ rf magnetic field. This connects the $|1,-1\rangle$ state to the $|2,1\rangle$ state via an intermediate virtual state with a detuning of $2.2 \mathrm{MHz}$ from the $|2,0\rangle$ state . The two-photon Rabi frequency is $600 \mathrm{~Hz}$, which is much faster than the characteristic frequency $2 \nu_{z}=96 \mathrm{~Hz}$ for the condensate to change shape.

We are able to view either component of a mixture of $|1,-1\rangle$ and $|2,1\rangle$ condensates or the combined density distribution. Observation of the atoms is a destructive measurement in which the TOP trap is suddenly turned off and the condensate is left to expand ballistically for $22 \mathrm{~ms}$. To image the combined density distribution of both states, a short pulse of "repump" light pumps the atoms from the $|1,-1\rangle$ state into the $F=2$ manifold. About $100 \mu s$ later, a $\sigma^{+}$circularly polarized probe beam $17 \mathrm{MHz}$ detuned from the $5 S_{1 / 2} F=2$ to $5 P_{3 / 2} F^{\prime}=3$ cycling transition is scattered by the atoms and the shadow is imaged onto a charge-coupled device camera. Imaging only the $|2,1\rangle$ condensate uses the same procedure, except that the pulse of repump light is omitted. Atoms in the $|1,-1\rangle$ state are far $(6.8 \mathrm{GHz})$ from resonance and invisible to the probe beam. Imaging only the $|1,-1\rangle$ atoms is similar to viewing both species simultaneously, but the $|2,1\rangle$ atoms are first "blown away" from the imaging region by a $2 \mathrm{~ms}, 60 \mu \mathrm{W} / \mathrm{cm}^{2}$ pulse of $F=2$ to $F^{\prime}=3$ light applied near the end of the ballistic expansion. This light has no effect on the $|1,-1\rangle$ atoms, to which a subsequent pulse of repump light is applied, pumping them into the $F=2$ manifold for probing. In Fig. 1p we show the population of each state as a func-

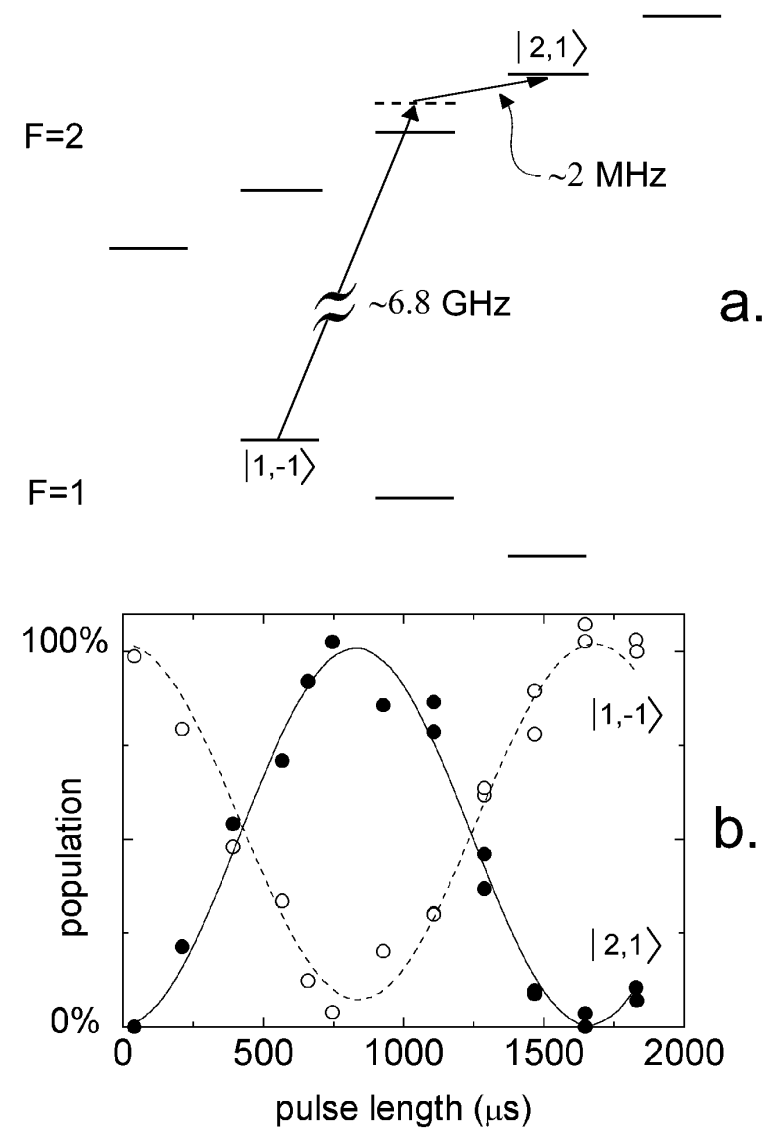

FIG. 1. (a). A diagram of the ground state hyperfine levels $(F=1,2)$ of ${ }^{87} \mathrm{Rb}$ shown with Zeeman splitting due to the presence of a magnetic field. The two-photon transition is driven between the $|1,-1\rangle$ and $|2,1\rangle$ states. (b) The Rabi oscillation of population between the $|1,-1\rangle$ (open circles) and $|2,1\rangle$ states (solid circles) as a function of the two-photon drive duration. The lines are fit to the data and show the expected sinusoidal oscillation.

tion of the two-photon pulse length. The Rabi oscillations have nearly $100 \%$ contrast, indicating that we are able to put any desired fraction into the $|2,1\rangle$ state.

We have investigated the dynamical behavior of the condensate after more than $99.5 \%$ of the atoms are transferred from the $|1,-1\rangle$ state to the $|2,1\rangle$ state. After the transfer, the condensate is allowed to evolve in the trap for a time interval $T$ before the cloud is released from the trap and probed. The experiment is repeated for different values of $T$, and the axial and radial widths of the condensate are measured using a fit to the twodimensional condensate image [21]. Fig. 2 illustrates the time dependence of both the axial and radial size of the atom cloud. Qualitatively, the data for both dimensions are consistent with a "compression" oscillation. The condensate shrinks for very early times, indicating a weaker 

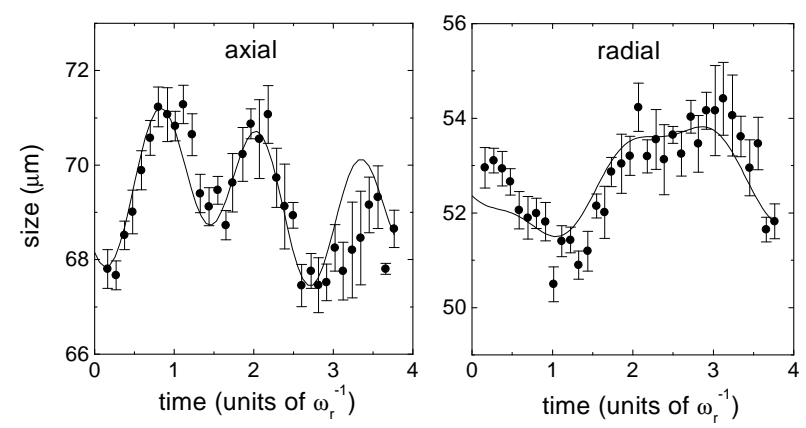

FIG. 2. Oscillation in the width of the cloud in both the axial and radial direction due to the instantaneous change in scattering length. The widths are for condensates as a function of free evolution time in units of the radial trap period $\left(\omega_{r}^{-1}=9.4 \mathrm{~ms}\right)$, followed by $22 \mathrm{~ms}$ of ballistic expansion. Each point is the average of approximately 10 measurements. Note that the fractional change in width is quite small. The solid line is a fit of the model to the data, with only the amplitude of the oscillation and the initial size as free parameters.

mean-field repulsion in the $|2,1\rangle$ state.

In order to describe the response to the change in scattering length, we use the Gross-Pitaevskii (GP) equation for the condensate wave function, with a time-dependent interaction term:

$$
i \hbar \frac{\partial \Phi(\mathbf{r}, t)}{\partial t}=\left[-\frac{\hbar^{2} \nabla^{2}}{2 m}+V(\mathbf{r})+U(t)|\Phi(\mathbf{r}, t)|^{2}\right] \Phi(\mathbf{r}, t)
$$

where $V(\mathbf{r})$ is the confining potential, $m$ is the ${ }^{87} \mathrm{Rb}$ mass, and $U(t)=4 \pi \hbar^{2} a(t) / m$. This has the form of a nonlinear Schrödinger equation, with a mean-field characterized by the $s$-wave scattering length $a(t)$. For $t<0, a(t)=a_{1}$, the scattering length for $|1,-1\rangle$ atoms on $|1,-1\rangle$ atoms. For $t>0, a(t)=a_{2}$, the $|2,1\rangle$ on $|2,1\rangle$ scattering length. The condensate is formed in its ground-state in which the density profile $n(\mathbf{r})=|\Phi(\mathbf{r})|^{2}$ is constant in time, and with the axial and radial widths $\left(w_{z}, w_{r}\right)$ determined by $V(\mathbf{r}), a_{1}$, and the number of atoms $N$. After a sudden change in $a(t), \Phi(\mathbf{r}, t)$ is projected onto a coherent superposition of its new ground-state and collective vibrational modes. Instead of solving the complete GP equation, one can use the Thomas-Fermi (TF) approximation, which corresponds to neglecting the quantum pressure term in the kinetic energy of the condensate. In this case, one can replace the GP equation with a pair of scalar equations of motion for the condensate widths [10] 12]:

$$
\ddot{w}_{i}+\left(2 \pi \nu_{i}\right)^{2} w_{i}-\left(\frac{15 \hbar^{2} N a(t)}{7^{5 / 2} m^{2}}\right) \frac{1}{w_{r}^{2} w_{z} w_{i}}=0 .
$$

Here the widths $w_{r}=\sqrt{\left\langle r^{2}\right\rangle}$ and $w_{z}=\sqrt{\left\langle z^{2}\right\rangle}$ are the radial and axial rms radii, and the subscript $i$ is to be replaced with either $r$ or $z$ for the respective widths. The TF approximation holds when $N$ is large [22] and, in this limit, the GP theory coincides with the hydrodynamic theory of superfluids [23].

Time-dependent behavior is predicted by numerically integrating Eqs. (2). The initial conditions $(t<0)$ are that the widths are at the values determined by the ground-state solution, $\dot{w}_{r}=\dot{w}_{z}=0$, and $a(t)=a_{1}$. After $t=0, a(t)=a_{2}$ and the integration proceeds for time $T$. We model the removal of the trap by setting $\nu_{r}=\nu_{z}=0$ and continue the numerical integration for the $22 \mathrm{~ms}$ free expansion. The axial and radial widths subsequently expand with different speeds. In the TF approximation, the ratio between the two expanded widths, averaged over $T$, can be shown to depend only on $\nu_{r}, \nu_{z}$ and the expansion time [12]. In our case, the theory predicts $w_{z} / w_{r}=1.29$, in good agreement with the measured value $w_{z} / w_{r}=1.31$. The oscillations of the widths correspond to a superposition of two $m=0$ modes. Since no angular momentum is imparted to the condensate by the change of scattering length, these are the only modes excited. The calculated mode frequencies are $\nu / \nu_{r}=1.80$ and $\nu / \nu_{r}=4.99$, and turn out to be independent of the amplitude in the range considered here. The two modes contribute to the oscillations in $z$ and $r$ with a different phase and amplitude, the axial motion behaving mainly as the fast mode and the radial motion as the slow one.

In each direction, the ratio of the amplitudes of the two modes is predicted to be constant over the range of possible scattering lengths relevant to the experiment. Thus, only the initial size and an overall amplitude are used as adjustable parameters in comparison of theory with experiment. The solid lines in Fig. 2 show the theoretical prediction using the best fit value of the oscillation amplitude, which is related to the scattering length ratio $a_{1} / a_{2}$. The predicted oscillations agree remarkably well with the shape of the data, for both the frequency and the phase of the two modes. From the fit amplitude we obtain the ratio of the scattering lengths $a_{1} / a_{2}=1.062 \pm 12$, which is consistent with the ratio of $1.059_{-6}^{+4}$ obtained in a theoretical calculation of binary collision parameters 24].

Analysis of the data is complicated slightly by an observed systematic dependence of the oscillation amplitude on the magnitude of the rf drive during the twophoton pulse. We believe that this effect is partly due to a small dressing of the atoms to the $|1,0\rangle$ or $|2,0\rangle$ state by the rf, and partly due to a coupling of the rf onto electronics controlling the trap potential. Both result in a change in strength of the confining potential during the two-photon pulse which returns to normal when the pulse is complete. After this impulsive perturbation, the BEC can be thought of as freely evolving with an initial "velocity" in the width $\left(\dot{w}_{r}, \dot{w}_{z} \neq 0\right)$. This is in contrast to the discrete change in scattering length, in which the BEC width has an initial offset from the equilibrium width, but no initial velocity. The systematic is manifested as 

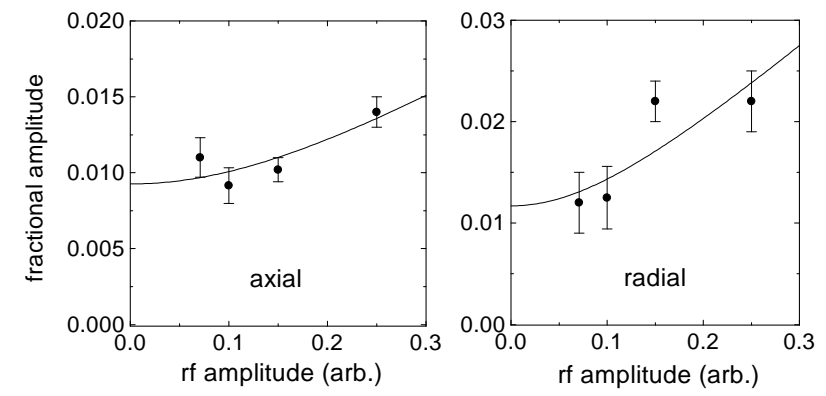

FIG. 3. Dependence of the oscillation amplitude on the rf strength in the two-photon transition. The vertical axis represents the fitted value of the fractional excitation amplitude, with error bars from uncertainty in the fit. The solid line shows extrapolation to zero rf amplitude.

an initial offset in the phase of the resulting oscillation, which is indeed observed for large rf amplitudes. Our result for the ratio was obtained by extrapolating to zero rf amplitude with a quadratic fit as shown in Fig. 3.

We have explored the dynamical response of a BoseEinstein condensate to a sudden change in the interaction strength. The agreement with the model is excellent, and presents a new and precise way of measuring a ratio of scattering lengths. This also allows a test of the theory for pressure shifts in atomic clocks [25]. Our method of creating two fully interpenetrating condensates will allow coherent control of the relative population and detailed examination of spatial and phase dynamics in the future.

We acknowledge useful conversations with the other members of the JILA BEC collaboration, and in particular with Chris Greene. This work is supported by the ONR, NSF, and NIST.

* Quantum physics division, National Institute of Standards and Technology.

[1] M.H. Anderson et al., Science 269, 198 (1995); K.B. Davis et al., Phys. Rev. Lett. 75, 3969 (1995); C.C. Bradley, C.A. Sackett, J.J. Tollett, and R.G. Hulet, Phys. Rev. Lett. 75, 1687 (1995); C.C. Bradley, C.A. Sackett, J.J. Tollett, R.G. Hulet, Phys. Rev. Lett. 79, 1170 (1997).

[2] M.-O. Mewes et al., Phys. Rev. Lett. 77, 416 (1996); M.J. Holland, D.S. Jin, M.L. Chiafalo, and J. Cooper, Phys. Rev. Lett. 78, 3801 (1997).

[3] D.S. Jin et al., Phys. Rev. Lett. 77, 420 (1996); M.-O. Mewes et al., Phys. Rev. Lett. 77, 988 (1996).

[4] J.R. Ensher et al., Phys. Rev. Lett. 77, 4984 (1996). See also S. Giorgini, L.P. Pitaevskii, and S. Stringari, Phys. Rev. Lett. 78, 3987 (1997).
[5] M.R. Andrews et al., Phys. Rev. Lett. 79, 553 (1997).

[6] C.C. Bradley, C.A. Sackett, and R.G. Hulet, Phys. Rev. Lett. 78, 985 (1997).

[7] E. Tiesinga, B.J. Verhaar, H.T.C. Stoof, Phys. Rev. A 47, 4114 (1993).

[8] P.O. Fedichev, Yu. Kagan, G.V. Shlyapnikov, and J.T.M Walraven, Phys. Rev. Lett. 77, 2913 (1996).

[9] John L. Bohn and P.S. Julienne, Phys. Rev. A 56, 1486 (1997).

[10] Yu. Kagan, E.L. Surkov, and G.V. Shlyapnikov, Phys. Rev. Lett. 79, 2604 (1997).

[11] Yu. Kagan, E.L. Surkov, G.V. Shlyapnikov, Phys. Rev. A 54, R1753 (1996) and Y. Castin and R. Dum, Phys. Rev. Lett. 77, 5315 (1996).

[12] F. Dalfovo, C. Minniti, S. Stringari and L. Pitaevskii, Phys. Lett. A 227, 259 (1997); F. Dalfovo, C. Minniti, and L. Pitaevskii, Phys. Rev. A 56, 4855 (1997).

[13] C.J. Myatt et al., Phys. Rev. Lett. 78, 586 (1997).

[14] P.S. Julienne, F.H. Mies, E. Tiesinga, and C.J. Williams, Phys. Rev. Lett. 78, 1880 (1997).

[15] James P. Burke, Jr., John L. Bohn, B.D. Esry, and Chris H. Greene, Phys. Rev. A 55, R2511 (1997).

[16] C.J. Myatt et al., Opt. Lett. 21, 290 (1996).

[17] C. Wieman, G. Flowers and S. Gilbert, Am. J. Phys. 63, 317 (1995). The getter and feedthroughs are in a small glass arm projecting from the glass MOT chamber. The rubidium vapor is readily turned on or off by controlling the current heating. We see no degradation of the trap lifetime compared to apparatus using a traditional (solid metallic) source. After initial seasoning the Rb is not readily consumed by the glass cell walls; a single $1 \mathrm{~cm}$ strip of getter material has shown no degradation after a year of nearly daily use.

[18] Wolfgang Petrich, Michael H. Anderson, Jason R. Ensher, and Eric A. Cornell, Phys. Rev. Lett. 74, 3352 (1995).

[19] D.S. Hall et al., Proc. SPIE 3270 (in press).

[20] John L. Bohn, (private communication).

[21] The expanded condensates are fit using a 2-dimensional projection of the Thomas-Fermi form for the density distribution in a harmonic trap. The fit also provides us with a measure of the number of atoms: we have measured a lifetime of $300 \mathrm{~ms}$ for the $|2,1\rangle$ state. This gives an upper limit on the $|2,1\rangle$ inelastic loss rate of $3 \times 10^{-13} \mathrm{~cm}^{3} / \mathrm{s}$. Number, and hence width is a decaying function of time, so we apply a small correction to the data (Fig. 2) based on this.

[22] Equations (2) can be indeed generalized to include effects beyond TF approximation. This has been done, for instance, by Pérez-García, Phys. Rev. Lett. 77, 5320 (1996). For the condensate used in the present work we have numerically checked that the corresponding changes in the final value of $a_{1} / a_{2}$ are much smaller than the experimental uncertainty.

[23] S. Stringari, Phys. Rev. Lett. 77, 2360 (1996)

[24] J.P. Burke, Jr. (private communication) based on methods in J.P. Burke, Jr., J.L. Bohn, B.D. Esry, and C.H. Greene, Phys. Rev. Lett. (in press); J.M Vogels et al., Phys. Rev. A 56, R1067 (1997).

[25] S.J.J.M.F. Kokkelmans, B.J. Verhaar, K. Gibble, D.J. Heinzen, Phys. Rev. A, 56, R4389, (1997). 